could be detected on the tonsils-usually confined to a small area at the top in between the angle formed by the anterior and posterior pillars of the fauces. Except in two instances, in which there was a very faint streak on each side of the midline, no blue coloration at all could be detected on the posterior wall of the pharynx. Subsequently it was found that Arnett (1943) had carried out a similar experiment using chewing-gum impregnated with gentian violet or methylene blue, and, like us, had observed little or no staining of the tonsils or posterior pharynx if the subject chewed his gum in the upright posture ; some staining, however, usually occurred if the recumbent position was adopted.

Thinking that our results might have been different if our subjects had not had healthy throats, we made observations on 6 patients in the isolation hospital who were suffering from measles or scarlet fever and whose tonsils were greatly enlarged. The pills were sucked in a semi-recumbent position, which, in view of Arnett's findings, may have affected the result. In one patient the whole of the medial surface of the tonsils was stained light green; in the remaining five the coloration was slight and was restricted to a small triangle at the top. In no instance was any coloration of the posterior pharyngeal wall seen.

These findings are in accordance with the observations of Bloomfield (1922), who concluded from an extensive series of experiments that there is a highly efficient mechanism, apparently dependent on suction currents, by which any bacteria introduced into the mouth are drawn directly backwards towards the oesophagus, avoiding the tonsils and posterior wall of the pharynx. It follows, therefore, that drugs given in the form of lozenges or chewing-gum can have little or no local effect on the throats of persons in the upright or sitting position unless they are sucked almost continuously throughout the day and night, as in MacGregor and Long's (1944) observations on penicillin pastilles. They are therefore unlikely to be of much value for prophylactic purposes in the ordinary healthy contact.

\section{Summary and Conclusions}

An outbreak of scarlet fever due to Str. pyogenes Type 11 in a residential preparatory school for boys, and an outbreak of sore throat accompanied by cases of scarlet fever due to Type 1 in a residential public school for girls, are briefly described.

Treatment of healthy and of convalescent carriers with 6 to 12 lozenges a day for 5 to 7 days, each lozenge containing $1 / 2$ gr. of sulphapyridine and $1 / 2 \mathrm{gr}$. of sulphathiazole, had no apparent effect, as compared with control carriers receiving either a formol spray and hypochlorite gargle or no treatment at all, in clearing the infecting lype of streptococcus from the throat.

Prophylactic treatment of healthy non-infected contacts with 6 lozenges a day had no apparent effect in preventing streptococcal infection of the throat.

Observations made on persons sucking methylene-blue tablets showed that only a fraction of the dye was deposited on the tonsils and none on the posterior pharyngeal wall; and it is therefore concluded that neither for the prophylaxis nor for the cure of streptococcal infection of the throat are lozenges containing sulphonamide drugs likely to be of value in practice.

It is maintained not that prophylactic sulphonamide treatment of streptococcal infections is useless but that further carefully controlled investigations should be carried out before this method, which is now being extensively used in the American Forces, is applied to the civilian or Service personnel of this country.

Our thanks are due to the medical officer of the girls' school, and to the head master of the boys' school, for their help in enabling us to carry out these investigations; and to Dr. Nora Archer, superintendent of the City Isolation Hospital, Oxford, for allowing us to make observations on patients with enlarged tonsils.

\section{REFERENCES}

Arnett, J. H. (1943). Amer. J. med. Sci., 205, 6.

Spink, W. W., Boynton, R., and Agnew, S. (1943). Proc. Soc. exp. Biol., N.Y., 52, 54.

Bloomfieid, A. L. (1922). Johns Hopk. Hosp. Bull. 33, 145.

Coburn, A. F. (1944).J. Amer. med. Ass., 126, 88.

Freis, E. D. (1944). Ibid., 126, 93.

Garson, P. (1943). British Medical Journal, 2, 452

Hamburger, M. (1944). J. infect. Dis., 75, 58, 71.

Hayden, A. F., and Bigger, J. W. (1945). British Medical Journal, 1, 81

Holbrook, W. P. (1944). J. Amer. med. Ass., 126, 84.

Kidd, P. (1944). Mon. Bull. Min. Hlth., Lond., 3, 135.

Topley, W. W. C., Greenwood, M., Wilson, J., and Newbold, E. M. (1928)

Topley, W. W. C., Greenwood,

Watson, R., F., Schwentker, F. F., Fetherston, J. E., and Rothbard, S. (1943).

J. Amer. med. Ass., 122, 730

Wright, J. (1945). British Medicai Journal. 1, 285.

\section{SURGERY AND ANAESTHESIA OF WAR WOUNDS OF THE ABDOMEN}

\author{
BY
}

\author{
S. O. AYLETT, F.R.C.S. \\ Major, R.A.M.C. \\ AND
}

A. F. ALSOP, B.Ch.

Major, R.A.M.C.

This paper includes a report of a series of missile wounds of the abdomen treated in the forward areas during the present campaign in Europe. In 200 consecutive battle casualties the abdomen was explored in 56 cases, so that a little over a quarter of the work of the forward surgeon is likely to be concerned with this type of injury. No selection whatsoever has been exercised in the choice of patient, and with one exception every case seen in the pre-operative ward and diagnosed as an intraabdominal lesion has been operated upon. This one patient was so severely injured that in spite of suitable resuscitative measures he collapsed after the administration of $0.1 \mathrm{~g}$. of pentothal, and it was considered useless to continue. Every patient is now given his chance, because it has been found that what may well be considered a hopeless case sometimes confounds such an opinion.

The mortality rate in this series is therefore a fair representation of that occurring among all patients with abdominal injuries who arrive at the forward surgical centre working within a few miles of the fighting-line. There is probably a small additional mortality at the base hospitals, due to subsequent complications, but when evacuated down the line after anything up to three weeks the patients have been in good condition.

All save one of our cases have been operated on under pentothal anaesthesia alone, the exception receiving in addition a spinal analgesic. For the success of this somewhat exacting type of work under difficult circumstances the choice of the anaesthetist is equally as important as the choice of the surgeon. The forward anaesthetist should be the most skilled and experienced available, for the type of case with which he has to deal is the most difficult of all to anaesthetize. In view of forthcoming campaigns in other parts of the globe, no apology is necessary in pleading that wherever a forward surgeon is sent he should be accompanied by a high-grade anaesthetist if his work is to be a success and not marred by anaesthetic mortality.

\section{Diagnosis}

The diagnosis of the straightforward abdominal case is easy ; that of the dubious one extremely difficult-so much so that some cases will always provide such an element of doubt that an occasional negative laparotomy will be undertaken. The reduction of this number to a minimum is, however, of paramount importance, because, as so many of these cases have other and severe lesions, such a laparotomy lessens their chances of recovery. Especially is this so in chest injuries, which may closely simulate an intra-abdominal lesion. Let it be said that there is no one symptom or sign that is always present, no one single observation upon which reliance can always be placed, and a diagnosis must be made by a summation of the points in favour and the points against.

A high proportion of cases in which a hollow viscus is injured give a history of early vomiting, repeated at intervals. This is not like the vomiting of a severely shocked patient, which occurs often after the administration of the traditional cup of hot sweet tea, which few of them can assimilate, or during the period of their recovery from the shock. This vomiting in those injured in the abdomen occurs when shock is absent and when nothing has been taken by the mouth.

The site of the wound should be located, and any injury to the anterior or lateral abdominal wall must be considered to be perforating until proved otherwise. If evidence from other signs and symptoms is not conclusive, all such wounds must be explored and followed to the end of their course before deciding that the peritoneum is not perforated. It is quite useless to 
probe the wound, as the track is often obscured by the natural elasticity of the tissues, and exploration must be by careful dissection. No attention must be paid to the size of the skin wound. We have seen a through-and-through injury of the stomach in which the entry in the skin was pin-head in size.

Wounds of the buttocks and flanks must always be suspect, and a thorough abdominal examination carried out in all cases. But intra-abdominal lesions may be caused by missiles entering high up in the chest or low down in the thigh. Where a rectal injury is suspected or possible a finger examination must be carried out.

In examining the abdomen it should not be forgotten that many of these patients are admitted heavily morphinized and that the classical sign of an abdominal catastrophe will be obscured or even absent. In one case, for example, no local tenderness, guarding, or rigidity was present whatsoever. On the other hand, certain extra-abdominal lesions will give rise to signs simulating an intraperitoneal viscus injury, particularly in perforating wounds of the lower chest, in which it is essential to establish a correct diagnosis. Unnecessary laparotomy may very well just turn the scales against recovery.

Injuries involving considerable damage to the abdominal musculature will naturally give rise to tenderness and rigidity, as will also lesions in which the kidney is affected. But in such cases, although the abdomen is held in apparent guarding, it is sometimes possible, in between the rapid respiratory excursions, to palpate muscular relaxation. This is never possible when true rigidity, the result of an intra-abdominal lesion, exists. It is maintained throughout the periods of respiration.

In spite of the sometimes dubious findings, full abdominal examination is of the highest importance, not only in determining a diagnosis but also as an indication of the best approach that can be made to deal with the condition. Auscultation of the abdomen must never be omitted, and we regard the information thus obtained as of the greatest importance. In the examination of a large number of patients suffering from wounds other than those involving the abdomen we have found that peristaltic sounds of the character heard in a normal man are usually present. In most of the cases in which there is a lesion of the bowel the sounds have been completely absent or reduced to such an extent that a long wait is necessary before a characteristic tinkle can be heard. Where a solid viscus is injured or where intraperitoneal bleeding alone is taking place, peristalsis in the early stages is present, though markedly reduced, before finally disappearing altogether.

In cases in which a retroperitoneal haematoma exists peristalsis is also reduced, and in the later stages when ileus sets in is absent altogether. Operation in these cases is contraindicated, and a differential diagnosis between such an injury and an intraperitoneal haemorrhage has to be established by consideration of the other diagnostic features. In the case of the latter injury, abdominal tenderness and shock are usually far greater. Location of the foreign body by radiographs is a valuable adjuvant in cases such as this, because an indication is given of what structures are likely to be damaged by the missile between the entrance wound and its final position.

\section{Resuscitation}

Not all cases of intraperitoneal injury need resuscitatory methods. The amount of transfusion required to render the patient fit for operation must vary according to the severity of the local as well as of the associated injuries. One patient, with a severe fracture of the femur running into the kneejoint in addition to the abdominal lesion, required 7 pints of blood before he was in any shape for operation. We have, however, aimed at improving the blood pressure to a minimum of $60 \mathrm{~mm}$. diastolic and $100 \mathrm{~mm}$. systolic. The mortality in cases presenting less than these figures has been high, although successes can be recorded among this group. Particularly is this so in patients in whom the injury has produced a large evisceration of the gut. Such patients do not respond well to resuscitation, but improve immediately the bowel is returned into the abdomen.

Pre-operative transfusion with whole blood was used in the majority of cases. A transfusion with either plasma or blood was invariably given during operation.

\section{Anaesthesia}

Sodium Pentothal.-Except in one case it was decided to use pentothal as the sole anaesthetic, not because it was believed in the beginning to be the best, but because it was the best drug available under the field conditions. The other drugs supplied were ether and chloroform, and (for spinal analgesia) stovaine $5 \%$ and light nupercaine.

Ether.-While ether causes little damage to healthy tissues and is rapidly excreted, it might well do considerable harm to tissues suffering from anoxaemia due to haemorrhage and shock, and its excretion is certainly prolonged where there is a much diminished circulatory volume.

Chioroform.-All that has been said of ether is applicable to chloroform, only to a greater degree, except that it is also harmful to healthy tissues.

Spinal Analgesia.-Enough has already been written by others on its dangers in severely shocked patients; it was used in one case, and will be referred to later, together with the reason for its use.

Splanchnic Analgesia.-This is ideal in cases of shock, but takes too long to administer to be considered under the conditions which pertained. The method to be described was first used by one of us (A. F. A.) in the Western Desert Winter Campaign, 1941-2 (7 cases), and again in Normandy, June. 1944 (5 cases).

Pre-operative Drugs.-As all the patients had had morphine at some time or times between their being wounded and their arriving at the surgical centre, and as it was often impossible to find out even approximately how much they had been given, they received no further drugs, sedative or otherwise, before operation.

\section{Pre-operative Method}

A $5 \%$ solution of pentothal was used, and, provided no crystallization had occurred, three-day-old solutions were found to be as efficacious as those that had been recently prepared. The solution should be kept in an air-tight container.

An estimate of the patient's condition was made, based chiefly on the diastolic blood pressure: as this decreased so the degree of shock was found to have increased. A diastolic pressure of below $70 \mathrm{~mm}$. $\mathrm{Hg}$ meant that at least a moderate degree of shock was present; to patients with diastolic pressure at these levels it was found unnecessary, and indeed dangerous, to give more than from 0.1 to $0.25 \mathrm{~g}$. as an initial dose ; this was usually found sufficient to enable the patient to have his clothes removed, his abdomen washed, to be shaved, and to be catheterized. If a greater amount was needed, not more than $0.2 \mathrm{~g}$. was given at a time, and a waiting period of at least two minutes was observed between doses in order to watch its effect. Of course, for slightly shocked patients far greater doses were required, and these could be given safely; to these patients as much as $1 \mathrm{~g}$. often had to be given for the preparations described.

After sufficient relaxation had been obtained for the peritoneum to be opened it was found that this relaxation was maintained for long periods-in some cases up to one hour -without further doses being required, although the conjunctival and cough reflexes were present. Sometimes small doses had to be given during the operation, and most cases had a further small dose immediately before closure of the peritoneum. As there was always a transfusion in progress during the operation, the pentothal was injected into the rubber tubing 1 in. to 3 in. from the transfusion needle.

No artificial airway was used unless there was respiratory obstruction, as this caused coughing in patients lightly anaesthetized. If one were necessary, either a nasopharyngea! tube or a Magill's endotracheal tube was passed.

\section{Difficulties Arising during Operation}

Respiratory Depression.-Early in the series there were several cases in which this happened to a severe degree. They were all cases in which more than $0.2 \mathrm{~g}$. had been given as a single dose in a matter of seconds. After these had occurred not more than $0.2 \mathrm{~g}$. was given to patients suffering from more than slight shock without waiting at least two minutes to observe its effect, and no more trouble in this respect was 
experienced. These cases were dealt with by passing a Magill's endotracheal tube and inflating the lungs with oxygen or air. There were no untoward consequences.

Vomiting.-This occurred occasionally, and, when it did, always in the period of preparation while the patient was lightly anaesthetized. A stomach-tube was then passed: this caused a temporary deterioration in the patient's condition, but recovery took place within a short time of the finish of the procedure. Regurgitation of fluid at any time during the operation without any effort being made by the patient happened in a few cases; this was dealt with by swabbing out the pharynx and passing a large (size 10) Magill's endotracheal tube.

Hiccup.-This came on only in cases that required large doses of pentothal-i.e., in the neighbourhood of $2 \mathrm{~g}$. in the first three-quarters of an hour and after the peritoneum had been opened. While the patient did not suffer the surgeon sometimes did, as it was inconvenient, when attempting to control bleeding in the renal or splenic pedicle, to have the diaphragm making spasmodic descents. This could be stopped in a few minutes by rebreathing oxygen without carbon dioxide absorption.

\section{Post-operative Drugs}

None were given unless the patient was restless, in which case morphine $1 / 6 \mathrm{gr}$. was administered intravenously-by this method because it was found that with established shock of any severity it was quite evident that, given by any other route, it was absorbed so slowly as to be of little use. To illustrate this, one case was, unknown to us, given morphine $1 / 2 \mathrm{gr}$., in two doses of $1 / 4$ gr., within 4 hours, on account of restlessness. It had no effect whatsoever. Now, this patient was practically pulseless when he left the theatre, and although he regained full consciousness he remained in an extreme state of shock until he died 48 hours later. It was quite evident that the morphine had not been taken into the circulation.

\section{Post-operative Complications}

Vomiting.-This occurred in one patient only. In this case vomiting took place four times in the 12 hours immediately after operation, and then ceased.

Pulmonary.-There was one case of bilateral basal collapse, which made a complete recovery, and three cases of unilateral pneumonia involving the bases only, one of them ending fatally. This patient had suffered from severe exposure, including immersion in the Rhine, after being wounded.

Deaths.-Out of 21 deaths, in 6 only could the anaesthetic be held to have played any part; of these 6,5 did not regain consciousness, and 1, already referred to, developed pneumonia. The 5 patients were all severely wounded men, 1 with severe additional injuries and 2 with peritonitis. One patient would undoubtedly have recovered consciousness except for the occurrence of a volvulus after operation.

\section{Discussion on Anaesthesia}

With this form of anaesthesia no great improvement in the ultimate recovery rate of perforating wounds of the abdomen can be claimed, but at least the mortality is no greater than with other forms. There are many advantages, such as: an abdominal relaxation equal to that obtained with spinal analgesia ; an almost complete absence of post-operative vomiting, which makes the work of a hard-pressed nursing staff during a battle considerably lighter; and a generally quiet recovery period in which the discomforts of the patient are not added to by the toxic effects of the anaesthetic. Chest complications are not prominent, and are certainly not more than is to be expected in a series of severe upper abdominal operations.

In the case requiring large doses of pentothal-that is, in the case.suffering little from shock-there is only a small margin between the levels of anaesthesia when the patient is sufficiently anaesthetized and when respiration ceases. Also, it is in these cases that hiccup is prone to occur; for this type spinal analgesia is recommended. It is of advantage to have the patient breathing oxygen in a closed circuit with carbon dioxide absorption; then if an overdose is inadvertently given it is a simple matter to keep respiration going by squeezing the rebreathing bag until physiological respiration is resumed. It is safest, however, to follow the method advised and to give small doses, repeated when required.

There are no cardiac disturbances, and such falls in blood pressure as occur can always be accounted for by the duration and severity of the operation. In one patient the blood pressure actually rose from $120 / 50$ before to $132 / 78$ after operation. Although in this series large doses of a barbiturate were being given there were no mental disturbances. One patient was unconscious for 12 hours; the remainder regained full consciousness within 6 hours.

It was indicative of extreme shock if the patient required $0.25 \mathrm{~g}$. or less during the period of preparation, usually lasting 10 to 15 minutes, and those patients requiring $1 \mathrm{~g}$. or less for the whole operation, including the preparatory period, stood a very poor chance of survival-one patient out of eight surviving.

\section{Operative Procedure}

Catheterization before the operation is begun is essential, as not only does this eliminate a possible mechanical obstruction but it will sometimes reveal unexpected renal damage. Preoperative stomach intubation has been limited to those cases in which vomiting has been marked and profuse, and, where necessary, it is now carried out under the initial stages of the anaesthetic.

With few exceptions a median or paramedian incision has been used, the site of choice being that on which it is thought maximum injury to the abdominal structures is present. Where the abdominal wall has been grossly injured, operation has been carried out through the existing wound, after its excision and, if necessary, enlargement.

In a thoraco-abdominal wound a decision has to be made as to whether the approach shall be through the diaphragm or by an abdominal incision. Where it has been decided during the diagnosis - in which radiographs are extremely valuable - that the damage is confined to structures easily approachable through the diaphragm, then this is the preferable route. Otherwise an alternative approach must be employed. It is only in wounds of the lower chest wall that the former approach is likely to be used, and it seems unnecessary to resect a rib, sufficient exposure being obtained by suitable retractors after an intercostal incision which excises the wound of entry. It should be noted in passing that splenectomy, through this approach. is a much simplified and more rapidly performed operation than through an abdominal incision.

In many cases the abdomen is filled with blood, which should be sucked and swabbed away before proceeding to a full inspection of the abdominal contents. This must be carried out in all cases save those in which the approach has been through the diaphragm and the surgeon is certain of the location of the injured structures. In some of our cases unexpected injury of the jejunum has been located when the main lesion has been far away in the right iliac fossa.

Some definite plan must be carried out in the examination of the abdomen; we have, as an initial stage, adopted the procedure of inspecting the region believed to have been damaged, and after dealing with the injury there, the remaining contents-the small intestine from the caecum to the duodenum, the large bowel from the transverse colon, down on either side -are searched. Criticism may be levelled at dealing with soiled gut first, in that spread of infection is likely, but against this may be placed the fact that if a perforation is not sealed at an early stage in the operation the actual manipulations of the gut encourage extensive leakage through the site of injury.

No intraperitoneal medication has been used in the series of cases reported, but we befieve that microcrystalline sulphadiazine is of value. It is probably best to leave a small catheter protruding from the peritoneal cavity, and through this to instil $5 \mathrm{~g}$. of the drug in suspension at 8-hourly intervals for 24 hours. To prevent leakage the tube is clipped at the end of each instillation.

Drainage of the general peritoneal cavity has been used only in cases of gross liver damage or those in which frankly infected peritoneal exudate was present. 


\section{Individual Injuries}

\section{Stomach}

Little need be said about these except to remark that wherever the anterior stomach wall has been perforated it is essential to open the lesser sac of peritoneum in order that the posterior wall may be examined.

\section{Duodenum}

Large wounds of the duodenum have a marked tendency to break down and to give rise to a duodenal fistula, from which the patient will ultimately die. In addition to suture of any large rent, a gastroenterostomy, performed at the time of the initial operation, is probably advisable. Drainage down to the site of the lesion should be instituted.

\section{Small Intestine}

It is often stated that the whole of the intestine should be examined before a decision is made whether resection or suture is to be carried out. This in practice is not satisfactory, as it is impossible, with multiple perforations, to prevent leakage from these while the rest of the bowel is examined. Unless, as the peritoneum is opened and the examination of the abdominal contents has begun, it is quite obvious that resection will be necessary, perforations should be treated as they present, either by invagination or by one or two through-and-through sutures.

Occasionally it will be necessary to resect a section of the bowel, and some of these repaired perforations may be included in the length of gut removed. Even so, the short period of time spent in the repair has not been wasted, as it has prevented the escape of intestinal contents and consequent soiling.

Resection should be carried out only in those cases in which the mesentery is so damaged as to interfere with the viability of the gut, or in injuries the local repair of which is likely to be followed by stenosis of the intestinal lumen. In our series, resection was carried out as the only intra-abdominal operation in 3 cases, of which two recovered, and, in addition to other procedures, on a further 3, two of which terminated fatally. Post-operative convalescence in these cases has caused little more anxiety than in those in which the lesion has been oversewn. The fatal results occurred in patients with severe associated lesions who were in extremely poor condition before operation. End-to-end anastomosis has been employed, the bowel being severed somewhat obliquely in order to give a larger lumen after suture.

\section{Caecum}

When the lesion is small and on the peritoneal surface it is perfectly safe to oversew or invaginate the perforation, but when the rent is large and the caecal wall grossly bruised, exteriorization with partial repair to form a caecostomy is advisable. In cases in which the posterior wall, uncovered by peritoneum, is injured, the surface should be exteriorized and the rent left open or sutured, according to the severity of the wound. The caecum will be brought out through a separate muscle splitting or dividing incision.

\section{The Large Bowel, excluding the Rectum}

The general principle of mobilization and exteriorization of the injured viscus is one that must dominate treatment; but before such procedure is carried out the perforations in the bowel should be temporarily sutured to prevent escape of faeces during the manipulations.

In the case of the transverse or pelvic colon exteriorization is simple, and rarely is mobilization necessary, but in other parts of the large bowel not provided with a mesentery the procedure is more difficult. In a case of injury to the upper part of the ascending colon the whole of the bowel from the caecum to the hepatic flexure may require freeing if the bowel is to be brought to the surface without tension. The peritoneum along the outer border of the bowel is incised, after which the latter is gently stripped of its attachments to the posterior abdominal wall, until it is held only by the thin layer of tissue containing the blood supply. Gauze dissection is of great value in this manœuvre. Some cases with a loose posterior attachment will free easily, but when the bowel is fixed fairly firmly, and the patient is broad and muscular, every possible band of tissue attachment has to be divided to obtain the necessary mobilization. A similar procedure is necessary for lesions of the descending colon, the injured bowel being brought out through a separate incision in the loin.

The type of colostomy performed is a matter of choice. Where it is possible, the ascending and descending links may be sutured together along the lines of a Paul-Mikulicz operation, the damaged portion of the bowel being subsequently cut away. An enterotome is used at a later stage to crush the spur intervening between the two loops of gut. It is, however, a procedure that is not always possible save in the case of the transverse and pelvic colons, and, where feasible, requires more extensive mobilization, the opening up of larger cellular spaces from which toxic absorption may occur, and additional time. We have not employed this technique, and have always exteriorized the bowel as a spur colostomy, using the method described by Ernest Miles. This is a matter of personal choice; but as a result of experience in civilian life and in periods spent at base hospitals we have found that the closure of such a colostomy is not a difficult procedure, and, provided that care is taken not to open the peritoneum, is unaccompanied by danger.

The argument advanced in favour of the exteriorization of all large-bowel wounds is that there is usually an associated bruising of the wall surrounding the actual perforation, and that any suture line is most likely to give way. This is undoubtedly true in most cases, especially when extensive faecal soiling is present. But it can never be denied that a colostomy, however temporary, is but a source of discomfort to the patient; particularly in the ascending colon, where the faecal discharge is fluid. If it can be avoided with safety a service has been done to the patient.

Occasionally the wound in the colon is small, and so clean-cut and without surrounding tissue damage that simple suture may be carried out, or the perforation sutured and extraperitonized by stitching the peritoneum and the rectus sheath to the bowel wall around the injury. This advocacy of any procedure other than exteriorization may be considered dangerous, but with prolonged experience of abdominal injuries selected cases can be treated safely in this fashion. Where the ascending colon has been exteriorized a short-circuit anastomosis between the small intestine and the transverse colon is of value in reducing the fluid faecal loss to a minimum.

In two of the cases in our series such extensive disruption of the colon was present as to necessitate resection with subsequent anastomosis. Both patients died, one after 16 days. This case was associated with a large injury of the duodenum, for which gastroenterostomy was performed 14 days after the injury. At the time of the second operation the anastomosis was observed to be firmly healed.

\section{The Rectum}

Where the lesion is intraperitoneal, repair and colostomy should be carried out. If the rupture is small and below the peritoneal reflection, divergence of the faecal flow by a colostomy is probably sufficient; but in cases in which the damage is severe, drainage of the pelvic faecal tissues is necessary, after removal of the coccyx and strapping up the rectum.

\section{Solid Viscus Injuries}

Wounds of the kidney may be present with other intra-abdominal lesions; when the organ is grossly disrupted, nephrectomy, through the abdominal route, must be carried out. Two cases in our series have been associated with such injuries; both terminated fatally.

\section{Splenectomy}

When any but the slightest damage has been suffered by the spleen, splenectomy is the correct treatment to adopt. We had three such cases; in two of them-in which injury to the viscus was the only damage-recovery resulted. The liver was damaged in only one case in this series, the rent being oversewn. This procedure is impossible when the injury is gross, or if it involves the diaphragmatic surface of the viscus and an abdominal approach has been made. Reliance must then be placed on packing, this being removed in 48 hours. When the injury is extensive and there is likely to be a considerable escape of bile, drainage of the area is desirable.

If, on the diagnostic points to which reference has been made, it san be assumed that the injury is likely to be on the diaphragmatic surface, approach through the chest is indicated.

\section{Post-operative Care}

Considerable controversy has arisen as to whether these patients should be nursed lying flat or in the typical Fowler position. Our own opinion is that, provided the patient is not sat up until his shock and its effects upon the circulation have disappeared, there is nothing to choose between these alternatives. Some patients who have been nursed lying down have begged to be allowed to sit up ; some sitting up have asked to lie down. It is the contrariness of human nature, as we do not believe that ultimate recovery or otherwise is affected thereby.

Gastric suction is instituted as soon as the patient recovers consciousness, the Ryle tube being passed through the nasal route. It is retained until only b.le is being drained from the stomach, and during this time fluids are allowed by mouth. So long as stomach suction is maintained-in the average case for from 3 to 4 days-four pints of saline and one of plasma are given by intravenous drip daily. Rarely, when suction 
has to be continued beyond this period in patients in whom the large bowel is not involved, saline may be administered through the rectal route. Provided a narrow catheter is used, and provided the flow is intermittent-a half-hour in every hour-absorption by this method is satisfactory. From time to time, after suction has ceased, the patient again starts to vomit, and there must then be no hesitation in resuming this and the saline drip.

Any severe ileus is rare in cases so treated, but in the event of abdominal distension becoming increasingly great, intravenous pituitrin-1 c.cm. in $10 \mathrm{c.cm}$. of water or saline-is of the utmost value, and the passage of flatus and faeces is immediate.

As soon as stomach suction is stopped the diet may rapidly be built up, the inclinations of the patient being largely followed. An enema or colostomy wash-out is useful when the bowels fail to act normally. Sulphaguanidine is valuable in reducing the offensiveness of colostomy.

A course of penicillin is now given as a routine, either by periodic injections or by a continuous intramuscular drip, as not only is the drug of value in counteracting the sepsis of the actual wound, but it has been found that staphylococci and streptococci are normal contaminants of the peritoneum in such cases. Those associated with grossly dirty wounds have been given sulphadiazine in the intravenous drip. In such cases alkali has always been administered by the same rqute.

Factors in the Causation of Death.-First and foremost in the causation of death, as in all civilian types of abdominal catastrophe, is the period of time elapsing between the onset of the lesion and the start of the operation; the accompanying Table illustrates this point. It will be seen that among all cases coming to operation within 10 hours of the receipt of the injury the mortality is $20 \%$, rising to $71 \%$ in those arriving after 20 hours.

Table showing Relation of Time of Wounding to Mortality Rate (54 Cases)

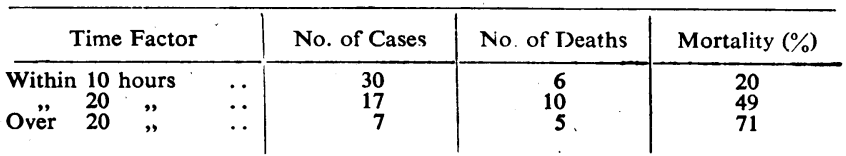

Coupled with this factor is the severity or otherwise of the lesion and associated extra-abdominal injuries. Of the six cases culminating fatally in the first group, five had gross and multiple injuries either inside the abdomen or associated with that lesion, and all died within 36 hours. The sixth patient died after operation for a post-operative obstruction.

\section{Summary and Conclusions}

The treatment of gunshot wounds of the abdomen is discussed. The use and advantages of pentothal anaesthesia in these cases are brought forward.

Pentothal has been found to be an ideal anaesthetic for abdominal surgery in shocked patients.

While this drug can be used with safety where there is only a mild degree of shock and where large doses have to be given, it is difficult of control and cannot be recommended in these cases.

The York Clinic at Guy's Hospital is the first psychiatric in-patient clinic to be established in this country as part of a general hospital in close association with the tradition of general medicine. It was not expected to be able to admit psychiatric patients till after the war, but the foresight of the York Council in proceeding with the building was justified, for at the end of 1943 the opening of the clinic for the purpose for which it was built was urgently requested by the Ministry of Health to provide beds for psychiatric casualties among officers of the Services. An immense amount of work had to be done by Dr. R. D. Gillespie (now Air Commodore, R.A.F.) and Mr. Lees Read to furnish and equip the clinic and prepare the organization. It opened on April 3, 1944, and a report on the first year's work has been issued as a cyclostyled document of six foolscap pages by the acting medical director, Dr. Felix W. Brown. There is undoubtedly a growing demand for treatment by modern psychiatric methods outside a mental hospital, and the York Clinic at Guy's has excellent opportunities to provide the right kind of training for future psychiatrists.

\section{THE BACTERIOLOGICAL EXAMINATION OF INFECTED DENTAL PULP CANALS AND USE OF PENICILLIN IN PULP-CANAL THERAPY

\author{
BY
}

FREDERICK E. SHAW, L.D.S.R.C.S.

London Hospital Medical College and Dental School Research Scholar

EVELYN SPRAWSON, M.C., D.Sc., M.R.C.S., L.D.S.

Professor of Dental Surgery, London Hospital Dental School AND

H. B. MAY, M.B., M.R.C.P.

Assistant Director, Clinical Laboratory, London Hospital

It has been shown by Bulleid (1938) and others that the commonest organism causing an infection of the pulp canal and periapical tissues is Str. viridans. Most strains of this organism are resistant to sulphonamides, especially in the presence of pus, but Fleming (1929) found that many are very sensitive to the action of penicillin, and therefore the effect of this chemotherapeutic agent on infected pulp canals seemed worthy of investigation.

Adams (1944) injected penicillin through the apical foramina in 2-c.cm. quantities, using 2,000 or 3,000 units for each injection. It is difficult to see how the periapical tissues could absorb this volume of fluid, and his criteria of sterility were not given; apparently an insufficient number of consecutive sterility results were accepted as adequate. He obtained a satisfactory result in all of 12 cases which he treated. It is surprising that in every case he apparently assumed that he was dealing with organisms sensitive to penicillin, although sensitivity tests were not carried out. In view of this work it was necessary to repeat the use of penicillin with more adequate bacteriological control.

It was clear that the common methods used in assessing the sterility of pulp canals-namely, $(a)$ the absence of clinical signs of periodontitis, and $(b)$ that the dressings removed appear clean and odourless-were inadequate for this work. Grossman (1936) showed that of 150 teeth, which by the appearance of the canal and odour of the dressing were judged by several dental practitioners to be ready for filling, $42 \%$ were still infected. A putrid odour may be produced by some organisms, especially anaerobic bacteria, but is often absent even in cases of gross infection by aerobic bacteria. A smear of the necrotic contents of the pulp canal, as suggested by Grossman (1940) is a very crude test of sterility. Radiographs of the periapical tissues may or may not reveal the presence of a rarefying osteitis due to infection, but this rarefaction must necessarily continue for some time even if the tissues have been rendered sterile ; thus radiographic evidence is of little value. The only methods of ascertaining whether sterility has been achieved that are capable of withstanding criticism by bacteriologists are cultural methods, and the technique should be one which will demonstrate the relative number of organisms and the presence of anaerobic as well as aerobic organisms, and must entail no extra effort or skill on the part of the dental surgeon.

The ability to sterilize the pulp canal and periapical tissues has long been disputed. Gottlieb et al. (1938), Thoma (1943), and Grossman (1940) state that these tissues can be sterilized; and Sprawson (1926) concludes that "when tested by cultures and films they can usually, after prolonged treatment, be rendered sterile," but he found that six months or more was necessary to obtain sterility in some .cases. Prinz (1911), Bulleid (1938), and others have stated that no antiseptic treatment will permanently sterilize a once-infected pulp canal. It is doubtful, however, if the bacteriological technique employed in all past work has been adequate to enable a final conclusion to be drawn.

Penicillin is a freely diffusible substance, and it was hoped that it would diffuse into the periapical tissues and increase their chance of permanent sterility.

\section{Method}

The following procedure was adopted for the bacteriological investigation of infected pulp canals and their treatment with penicillin. Single-rooted teeth only were investigated. On the 